\title{
On Current-Carrying Cosmic Strings and the Generalized Rainich Algebra in Scalar-Tensor Gravities
}

\author{
M. Leineker Costa, \\ Instituto de Física, Universidade de Brasília, CEP: 70910-900, Brasília, DF, Brazil \\ M. E. X. Guimarães, \\ Departamento de Matemática, Universidade de Brasília, CEP: 70910-900, Brasília, DF, Brazil \\ and A. L. Naves de Oliveira \\ Departamento de Física, Universidade Estadual de Londrina, Caixa Postal 6001, Londrina-Paraná, Brazil \\ (Received on 11 October, 2005)

\begin{abstract}
We obtain exact solutions for a static and charged cosmic string in a Einstein-Maxwell-Dilaton theory of a scalar-tensor type in $(3+1)$-Dimensions. This theory is specified by the dilaton field $\phi$, the graviton field $g_{\mu v}$ and the electromagnetic field $F_{\mu \nu}$, and one post-Newtonian parameter $\alpha(\phi)$. It contains three different cases, each of them corresponding to a particular solution of the Rainich algebra for the Ricci tensor.
\end{abstract}

\section{INTRODUCTION}

Current-carrying strings have been considered in the framework of Einstein's gravity. Here, we intend to generalize these results considering a scalar-tensor gravity. The motivation for this relies on the fact that, theoretically, the possibility that gravity might not be fundamentally Einsteinian is gathering credence. This is in part a consequence of superstring theory, which is consistent in ten dimensions (or M-theory in eleven dimensions), but also the more phenomenological recent developments of "braneworld" scenarios have motivated the study of other gravitational theories in four-dimensions. In any case, clearly, if gravity is essentially scalar-tensorial there will be direct implications on observed effects both in the small scale scenarios of alternative theories of gravity and in the large scale cosmological scenarios from modified gravity.

Our purpose in this work is to generalize previous results [1-4] and to study static and charged cosmic strings as particular solutions of a Einstein-Maxwell-Dilaton theory of a scalar-tensor type in (3+1)-dimensions by proposing a generalization of the Rainich algebra for the Ricci tensor. We obtain three exact solutions corresponding to an electric, magnetic and "mixed" strings. This manuscript is organized as follows. In the section 2, we review briefly the model for a currentcarrying cosmic string in the scalar-tensor theory. In section 3 , we generalize the Rainich algebra and we obtain the three exact solutions, each of them corresponding to the metric of a current-carrying string. In section 4 , we summarize our main results.

\section{CURRENT-CARRYING STRINGS}

In this section we will study the gravitational field generated by three cases of a string carrying a current. For technical purposes, we will work in the so-called conformal (Einstein) frame, in which the scalar and tensor degrees of freedom do not mix

$$
\mathcal{S}=\frac{1}{16 \pi G_{*}} \int d^{4} x \sqrt{-g}\left[R-2 \partial_{\mu} \phi \partial^{\mu} \phi\right]+\mathcal{S}_{m},
$$

where $\mathcal{S}_{m}$ is the action of matter. In what follows, we will contemplate configurations corresponding to an isolated, static current-carrying vortex lying in the $z$-axis., which arises from a spontaneous symmetry breaking of the $U(1) \times U_{\text {em }}(1)$ model with a potential suitably chosen in order that the pair $\left(\varphi, B_{\mu}\right)$ breaks one symmetry $U(1)$ in vacuum (given rise to the vortex configuration) and the second pair $\left(\sigma, A_{\mu}\right)$ breaks the symmetry $U_{e m}(1)$ in the core of the vortex (giving rise to the superconducting properties) [1-6].

Variation of the action (1) with respect to the metric $g_{\mu v}$ and to the dilaton field $\phi$ gives the modified Einstein's equations and the wave equation for the dilaton, respectively

$$
\begin{aligned}
G_{\mu v} & =2 \partial_{\mu} \phi \partial_{v} \phi-g_{\mu v} g^{\alpha \beta} \partial_{\alpha} \phi \partial_{\beta} \phi+8 \pi G_{*} T_{\mu v}, \\
\square_{g} \phi & =-4 \pi G_{*} \alpha(\phi) T .
\end{aligned}
$$

Here, $T_{\mu v}=\frac{-2}{\sqrt{-g}} \frac{\delta S_{m}}{\delta g_{\mu v}}$ is the energy-momentum tensor of the matter fields.

\section{THE EXTERIOR METRICS}

Due to the specific properties of the Maxwell tensor

$$
T_{\mu}^{\mu}=0 \text { and } T_{v}{ }^{\alpha} T_{\alpha}{ }^{\mu}=\frac{1}{4}\left(T^{\alpha \beta} T_{\alpha \beta}\right) \delta_{v}^{\mu},
$$

the Einstein's equations may be transformed into some algebraic relations called Rainich algebra [7] which in scalartensor theories are modified by a term which depends explicitly on the dilaton field

$$
\begin{array}{r}
R=R_{t}^{t}+R_{r}^{r}+R_{\theta}^{\theta}+R_{z}^{z}=2 g^{r r}\left(\phi^{\prime}\right)^{2}, \\
\left(R_{t}^{t}\right)^{2}=\left(R_{r}^{r}-2 g^{r r}\left(\phi^{\prime}\right)^{2}\right)^{2}=\left(R_{\theta}^{\theta}\right)^{2}=\left(R_{z}^{z}\right)^{2},
\end{array}
$$

we can see from (5) that there exist three possible solutions 
- Case I:

$$
R_{t}^{t}=-R_{\theta}^{\theta} \quad R_{t}^{t}=R_{r}^{r}-2 g^{r r}\left(\phi^{\prime}\right)^{2} \quad R_{\theta}^{\theta}=R_{z}^{z}
$$

- Case II:

$$
R_{t}^{t}=R_{\theta}^{\theta} \quad R_{t}^{t}=-R_{r}^{r}+2 g^{r r}\left(\phi^{\prime}\right)^{2} \quad R_{\theta}^{\theta}=-R_{z}^{z}
$$

- Case III:

$$
R_{t}^{t}=-R_{\theta}^{\theta} \quad R_{t}^{t}=-R_{r}^{r}+2 g^{r r}\left(\phi^{\prime}\right)^{2} \quad R_{\theta}^{\theta}=-R_{z}^{z}
$$

For each of these cases we find the following exterior metric, respectively

$$
\begin{aligned}
d s_{E}^{2}= & \left(\frac{r}{r_{0}}\right)^{2 m^{2}-2 n} W^{2}(r)\left(d r^{2}+d z^{2}\right) \\
+ & \left(\frac{r}{r_{0}}\right)^{-2 n} W^{2}(r) B^{2} r^{2} d \theta^{2} \\
& -\left(\frac{r}{r_{0}}\right)^{2 n} \frac{1}{W^{2}(r)} d t^{2} \\
d s_{M}^{2}= & \left(\frac{r}{r_{0}}\right)^{2 m^{2}-2 n} \\
+ & \left(\frac{r}{r_{0}}\right)^{-2 n} W^{2}(r)\left(d r^{2}-d t^{2}\right) \\
& +\left(\frac{r}{r_{0}}\right)^{2 n} \frac{1}{W^{2}(r)} d z^{2}, \\
d s_{M i}^{2}= & \left(\frac{r}{r_{0}}\right)^{2} \frac{V^{2}(r)}{(n-1)} d \theta^{2} \\
+ & \left(\frac{r}{r_{0}}\right)^{F+1} \frac{V^{2}(r)}{(n-1)}\left(d r^{2}-d t^{2}\right) \\
+ & B^{2} \frac{(n-1)}{V^{2}(r)} d \theta^{2},
\end{aligned}
$$

where

$$
\begin{aligned}
W(r) & =\frac{\left(\frac{r}{r_{0}}\right)^{2 n}+k}{1+k} \\
V(r) & =\left[-c_{1} \sin (\sqrt{n-1} \ln r)+c_{2} \cos (\sqrt{n-1} \ln r)\right] .
\end{aligned}
$$

All the integration constants are to be determined after the inclusion of matter fields.

\section{SUMMARY}

In this work we obtain exact solutions for a static and charged cosmic string in a Einstein-Maxwell-Dilaton theory of a scalar-tensor type in (3+1)-Dimensions. This theory is specified by the dilaton field $\phi$, the graviton field $g_{\mu \nu}$ and the electromagnetic field $F_{\mu \nu}$, and one post-Newtonian parameter $\alpha(\phi)$. It contains three different cases, each of them corresponding to a particular solution of the Rainich algebra for the Ricci tensor.

The three solutions correspond to solutions of an electric, magnetic and "mixed" strings. Each of them, stemming from the low-energy limit of the string theory, present very distinct gravitational features providing us with useful constraints on the underlying microscopic model $[8,9]$.

\section{Acknowledgements}

M. Leineker Costa would like to thank CAPES and FINATEC/UnB for a support. A. L. Naves de Oliveira and M. E. X. Guimarães would like to thank $\mathrm{CNPq}$ for a support.
[1] P. Peter and D. Puy, Phys. Rev. D 48, 5546 (1993).

[2] C. N. Ferreira, M. E. X. Guimarães and J. A. Helayel-Neto, Nucl. Phys. B 581, 165 (2000).

[3] A. L. N. Oliveira and M. E. X. Guimarães, Phys. Lett. A 311, 474 (2003).

[4] A. L. N. Oliveira and M. E. X. Guimarães, Phys. Rev. D67 , 123514 (2003).

[5] E. Witten, Nucl. Phys. B 557 (1985).

[6] B. Carter, in "Formation and Evolution of Cosmic Strings", ed. G.W. Gibbons, S. W. Hawking and T. Vachaspati (Cabridge Univ.
Press, Cambridge, 1990).

[7] L. Witten, in "Gravitation: an Introduction to Current Research, ed. L. Witten (Jonh Wiley and Sons, New York, 1962).

[8] M. Leineker Costa, A. L. Naves de Oliveira and M. E. X. Guimarães, "Contributions from Dilatonic Strings to the Flat Behaviour of the Rotational Curves in Galaxies", gr-qc/0507109.

[9] M. Leineker Costa, A. L. Naves de Oliveira and M. E. X. Guimarães, in preparation. 